\title{
Pancreas Infection, CTCAE
}

National Cancer Institute

\section{Source}

National Cancer Institute. Pancreas Infection, CT CAE. NCI Thesaurus. Code C143728.

A disorder characterized by an infectious process involving the pancreas. 